\title{
Detección molecular de Leishmania (Viannia) Iainsoni Silveira, Shaw, Braga \& Ishikawa, 1987 (Kinetoplastida: Trypanosomatidae) en humanos de Paraguay
}

\section{Detección molecular de Leishmania (Viannia) Iainsoni Silveira, Shaw, Braga \& Ishikawa, 1987 (Kinetoplastida: Trypanosomatidae) en humanos de Paraguay}

\author{
Irene R. Benítez' \\ Ricardo Andrade Zampieri ${ }^{2}$ \\ Ana Nilce Silveira Elkhoury ${ }^{3}$ \\ Lucile Maria Floeter-Winter ${ }^{2}$ \\ Jose Angelo Lauletta Lindoso ${ }^{4}$ \\ José Pereira' \\ Pier Cacciali ${ }^{5,6}$ \\ Mara Muñóz ${ }^{7}$ \\ Miguel Angel Aragón López ${ }^{7}$
}

1. Ministerio de Salud Pública y Bienestar Social. Programa Nacional Control Leishmaniasis, (SENEPA). Manuel Domínguez e/ Brasil, Asunción, Paraguay.

2. Universidade de São Paulo, Instituto de Biociências, Laboratorio de Fisiologia de Tripanossomatídeos, Departamento de Fisiologia. Rua do Matão, travessa 14, ํ․ 101, Cep: 05508-090, Cidade Universitária Brazil. 3. Pan American Health Organization. Communicable Diseases and Health Analysis, Duque de Caxias, Rio de Janeiro,Brazil.

4. Instituto de Medicina Tropical de São Paulo-USP, São Paulo, SP, Brazil.

5. Fundación ie, República de Colombia. Laboratorio de Desarrollo Ambiental. 1102, 1548.

Asunción, Paraguay.

6. Instituto de Investigación Biológica del Paraguay, Del Escudo 1607, 1425 Asunción, Paraguay.

7. OPS-OMS-Paraguay, Mariscal López 953, 1527 Asunción, Paraguay.

\section{Resumen}

La leishmaniasis es una de las enfermedades tropicales más desatendidas, causada por el parásito Leishmania. En Paraguay, la especie responsable de la leishmaniasis cutánea es (LC) es L. (Viannia) braziliensis. Aquí se reporta un caso diagnosticado de Leishmaniasis, y análisis moleculares empleando reacción en cadena de la polimerasa - polimorfismos de restricción de fragmentos de restricción (PCRRFLP) demostraron que el caso fue causado por $L$. (V.) lainsoni. Este es el primer registro de esta especie 
para Paraguay, con lo cual se extiende el rango de distribución conocido de la especie, unos $1.430 \mathrm{~km}$ al sur de localidades previamente conocidas. Se necesitan más estudios para conocer la incidencia real de esta especie en casos de LC en Paraguay, y para identificar reservorios naturales del parásito en la naturaleza.

Palabras clave: Epidemiología, Leishmaniasis, PCR-RFLP, Sudamérica

\begin{abstract}
Leishmaniasis is one of the most neglected tropical diseases worldwide caused by the parasite Leishmania. In Paraguay the species responsible for cutaneous leishmaniasis (CL) is $L$. (Viannia) braziliensis. Here we report a case diagnosed with Leishmaniasis, and molecular analyses using polymerase chain reaction-restriction fragment length polymorphism (PCR-RFLP) demonstrate that the case was caused by $L$. (V.) lainsoni. This is the first record of this species for Paraguay, with which we extend the distribution range of the parasite 1,430 km southwards from the southernmost previous known locality. More studies are needed to know the actual incidence of this species in cases of CL in Paraguay, and to identify natural reservoirs in the wild
\end{abstract}

Key words: Epidemiology, Leishmaniasis, PCR-RFLP, South America

Fecha de recepción: 01-04-2019

Fecha de aceptación: 14-04-2019

Correspondencia:

Dr. Irene R. Benítez

irenerocio@gmail.com

Programa Nacional Control Leishmaniasis (SENEPA)

\title{
Introducción
}

La leishmaniasis es considerada una de las enfermedades tropicales más desatendidas en el mundo entero ${ }^{(1)}$, y es transmitida por la picadura de hembras de flebótomos (comúnmente del género Lutzomyia: Psychodidae: Diptera) infectadas con el parásito del género Leishmania Ross, $1903^{(2)}$. Existen varias especies de Leishmania, las cuales están presentes en casi todos los continentes con excepción de Oceanía ${ }^{(3)}$. Respecto a las manifestaciones patológicas, se pueden diferenciar tres tipos principales de leishmaniasis (leishmaniasis visceral, y leishmaniasis tegumentaria que se divide en leishmaniasis cutánea y leishmaniasis mucocutánea) y todas estas están presentes en Paraguay ${ }^{(4,5)}$, y según los registros, la enfermedad ya lleva instalada en el país más de cien años ${ }^{(6)}$. La leishmaniasis cutánea (LC) es una infección crónica que muestra variación regional respecto al tipo de lesión dérmica producida por el parásito, aunque la más común es la úlcera moldurada ${ }^{(7)}$.

La especie de Leishmania registrada en Paraguay como responsable de la LC es oficialmente $L$. (Viannia) braziliensis ${ }^{(4,5,8)}$. Sin embargo, se registró un caso sospechoso causado por L. (V.) guyanensis ${ }^{(9)}$, y dos más recientes atribuidos a $L$. (Leishmania) infantum chagasi reconocidos mediante análisis moleculares con PCR ${ }^{(10)}$. La concentración más alta de casos de LC para Paraguay fue reportada en el este del país (Alto Paraná, Caaguazú, Canindeyú y San Pedro) ${ }^{(5)}$. El caso de LC causado por $L$. (L.) infantum 
chagasi fue registrado en la ciudad de Pedro Juan Caballero, limítrofe con Brasil, y el caso producido por L. (V.) guyanensis se reportó en el Departamento de San Pedro ${ }^{(9)}$.

En Paraguay, la vigilancia epidemiológica de leishmaniasis está a cargo del Programa Nacional de Control de la Leishmaniasis, dependiente del SENEPA (Servicio Nacional de Erradicación del Paludismo), perteneciente al MSPyBS (Ministerio de Salud Pública y Bienestar Social). Como parte de la vigilancia nacional rutinaria, y empleando un protocolo de análisis molecular, aquí se reporta el primer registro de Leishmania (Viannia) lainsoni Silveira, Shaw, Braga \& Ishikawa, 1987, la cual representa una especie adicional para el país

\section{Materiales y métodos}

Caso clínico: Mujer de 27 años de edad, ama de casa, oriunda del Departamento de San Pedro (Fig. 1). La paciente consultó en un servicio de atención médica (Hospital Regional de San Pedro), y fue posteriormente trasladada al SENEPA debido a la presencia de una lesión dérmica en el miembro inferior izquierdo, la cual inició dos meses antes como una pequeña pústula, con aumento gradual de tamaño y pérdida de sustancia. Algesia ocasional.

Figura 1. Distribución de Leishmania (Viannia) lainsoni (sombreado marrón) resaltando la localidad más al sur previamente conocida (punto rojo), y el nuevo registro para Paraguay (estrella negra) extendiendo aproximadamente $1,430 \mathrm{~km}$ al sur el rango de la especie.

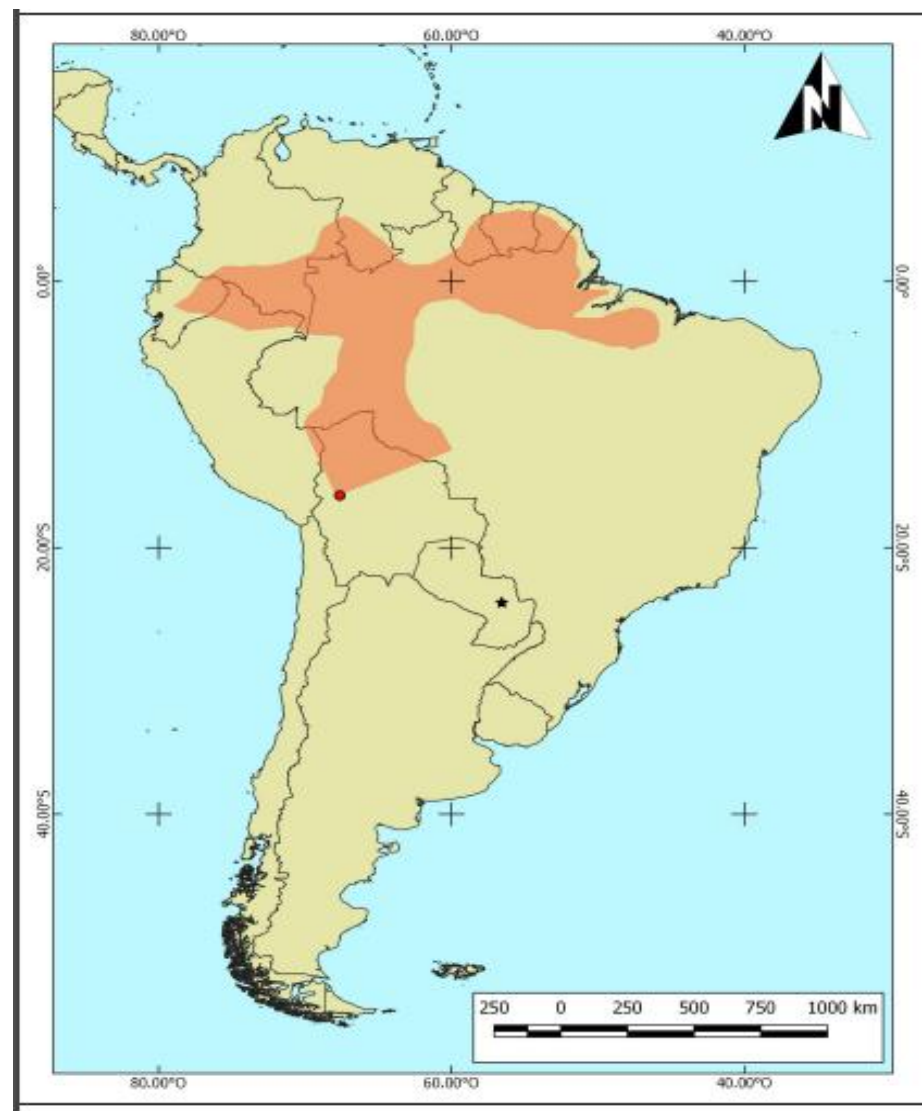

La paciente no presenta historia patológica relevante. La paciente declara no haber hecho traslados importantes ni locales ni internacionales.

El examen de la lesión, fue registrado como sigue: úlcera límpida con tejido granular y restos de fibra en la superficie, bordes moldurado eritematoso, levemente infiltrado, $8 \mathrm{~cm}$ de diámetro, localizada en el lado antero-externo de la región tibial izquierda. No se observan heridas mucosas.

Pruebas sanguíneas mostraron perfiles hepáticos y lipídicos dentro de los valores normales. 
Análisis microscópico: Siguiendo una diagnosis clínica para LC, se tomaron algunas muestras de la herida para estudios parasitológicos. Un frotis de tejido de la herida (tomada de una profundidad de $3 \mathrm{~mm}$ ) fue fijada en etanol $96 \%$. La muestra fue coloreada con el método de tinción Wright-Giemsa (WGSM) en una concentración de dos gotas por $\mathrm{ml}$ de agua destilada y dos gotas de buffer PBS (Phosphate-buffered saline) ( $\mathrm{pH}:$ 7.2), durante $30 \mathrm{~min}$. Este análisis fue hecho en el Laboratorio de Bacteriología del "Hospital Nacional de Itauguá".

Análisis moleculares: Tejido adicional fue extraído para análisis de ADN. Una muestra de tejido fue inmerso en $1 \mathrm{ml}$ de xylol e incubado a 80-90 ${ }^{\circ} \mathrm{C}$ por $5 \mathrm{~min}$. Luego, la muestra fue centrifugada a $13000 \mathrm{rpm}$ por $5 \mathrm{~min}$, para eliminar el exceso de solvente. Este paso fue repetido dos veces. La muestra fue posteriormente inmersa en $1 \mathrm{ml}$ de etanol 98\% e incubada a temperatura ambiente por 5 min, y centrifugada a $13000 \mathrm{rpm}$ por $5 \mathrm{~min}$. Inmediatamente el tejido fue secado a $50^{\circ} \mathrm{C}$ y el ADN extraído y purificado usando el kit DNeasey ${ }^{\circledast}$ de Qiagen ${ }^{\circledast}$.

Las reacciones de pre-amplificación fueron hechas usando el kit TopTaq Master Mix ${ }^{\circledR}$ de Qiagen ${ }^{\circledR}$ (el cual ya contiene dNTP's), adicionando $0,2 \mu \mathrm{M}$ de cada primer Forward (preamp_F: 5' -GGC ATC CTG AAC GTG TCC G- 3') y Reverse (preamp_R: 5' -ATC TTG GTC ATG ATC GGG TTG CAT- 3') para el gen de la proteína heat-shock 70 (hsp70) ${ }^{(11)}$, y 2,5 $\mu$ l de ADN purificado de la muestra, alcanzando un volumen final de $25 \mu$ l. Cabe mencionar que los primers utilizados aquí fueron diseñados para aumentar la sensibilidad, y son empleados por primera vez en este trabajo. Las condiciones de PCR fueron como sigue: desnaturalización inicial $5 \min \left(94^{\circ} \mathrm{C}\right)$ - [desnaturalización $30 \sec \left(94^{\circ} \mathrm{C}\right)$ - hibridación $30 \sec \left(55^{\circ} \mathrm{C}\right)$ - elongación $\left.30 \mathrm{sec}\left(72^{\circ} \mathrm{C}\right)\right] \times 30$. Para comparación, se incluyeron cepas de las siguientes especies de Leishmania como referencia: Leishmania (Leishmania) infantum, Leishmania (Leishmania) amazonensis, Leishmania (Leishmania) mexicana, Leishmania (Viannia) lainsoni, Leishmania (Viannia) braziliensis, Leishmania (Viannia) guyanensis, Leishmania (Viannia) naiffi y Leishmania (Viannia) shawi.

Análisis de PCR-RFLP fueron realizados usando 0,2 $\mu \mathrm{l}$ de los productos de la pre-amplificación, siguiendo el protocolo descrito por Graça et al. ${ }^{(12)}$, generando una secuencia de $234 \mathrm{bp}$, el cual fue tratado con enzimas de restricción (BstU I, Hae III y Mbo I). Esto generó perfiles de electroforesis, los cuales permitieron la comparación con las cepas de referencia de Leishmania. El gel de electrophoresis fue hecho con agarosa 3\% en buffer TAE buffer, y teñido con bromuro de etidio. La visualización fue hecha sobre transilluminator de luz UV, TFX-20M (Life Technologies, Carlsbad, Califórnia, USA) y fotografiado por un fotodocumentador LAB-020 (LabTrade do Brasil, Cotia, São Paulo, Brasil).

\section{Resultados}

El examen visual del tejido bajo aumento de 100x, se observaron estructuras ovales de 2-4 $\mu \mathrm{m}$, con un núcleo coloreado con tinción Wright-Giemsa; lo cual coincide con amastigotes de Leishmania sp.

El perfil de RFLP obtenido para la muestra de Paraguay en la electroforesis para BstU I mostró una banda compatible con L. (L.) infantum, L. (L.) amazonensis, L. (L.) mexicana y L. (V.) lainsoni (Fig. 2A). Cuando comparado con las especies de referencia, usando Hae Ill enzima, la muestra paraguaya coincide con L. (V.) lainsoni y L. (V.) shawi (Fig. 2B). Finalmente, cuando la muestra fue tratada con la enzima Mbo I, las bandas generadas coinciden con la mayoría de las cepas, con excepción de $L$. (V.) guyanensis y $L$. $(V$.) shawi (Fig. 2C). Por lo tanto, la comparación integrada muestra que $L$. (V.) lainsoni fue la única especie que coincidió con la muestra de Paraguay en los tres casos. Además, con el análisis combinado de los tres perfiles probados fue posible excluir la compatibilidad con las restantes especies usadas como referencia 


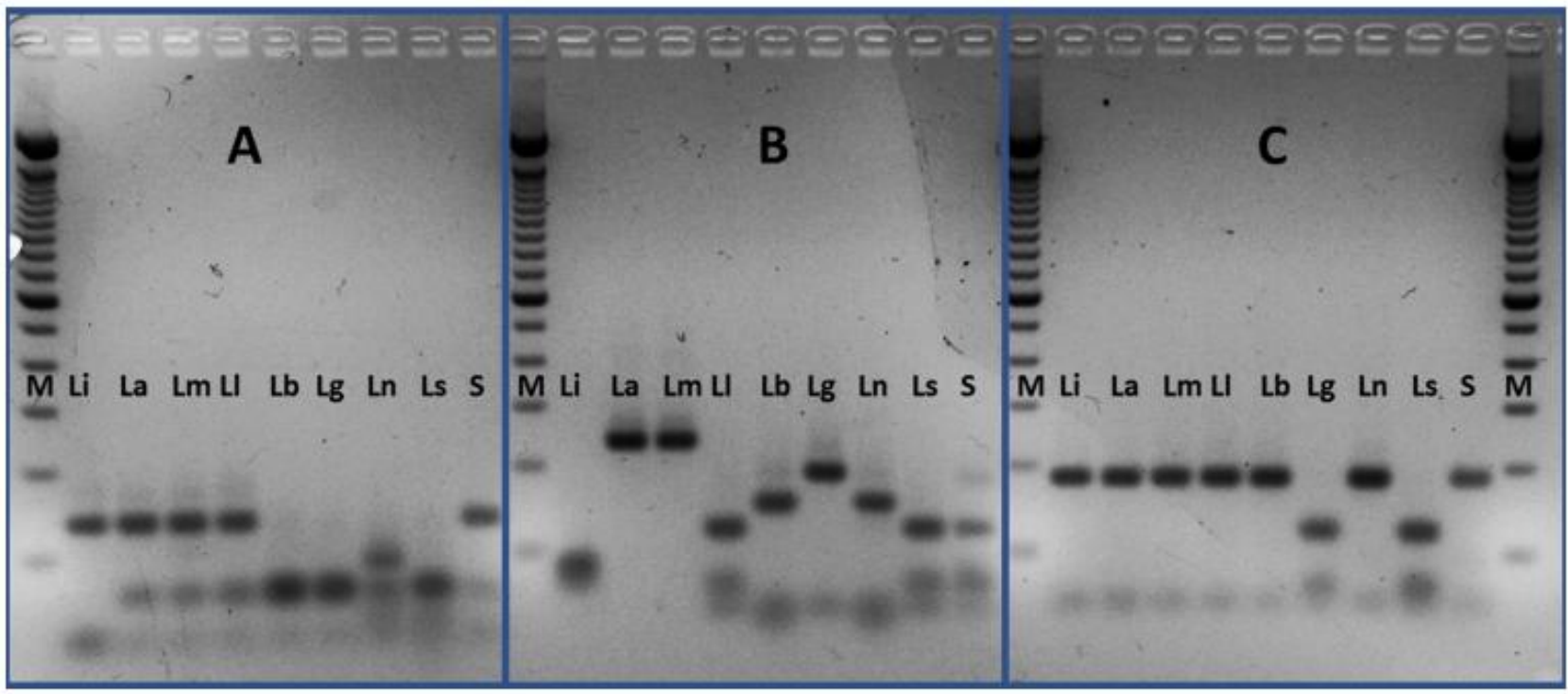

Figura 2. Perfiles de PCR-RFLP para el gen hsp70 usando enzimas de restricción BstU I (A), Hae III (B) y Mbo I (C). "M" indica las bandas de referencia (100 bp), y la muestra objetivo está especificada con una "S". Las cepas de las siguientes especies fueron usadas para comparación - Li: L. (L.) infantum, La: L. (L.) amazonensis, Lm: L. (L.) mexicana, LI: L. (V.) lainsoni, Lb: L. (V.) braziliensis, Lg: L. (V.) guyanensis, Ln: L. (V.) naiffi y Ls: L. (V.) shawi.

\section{Discusión}

La correcta identificación de un parásito es un factor clave para la diagnosis de una enfermedad y el subsecuente tratamiento médico ${ }^{(13)}$. Sin embargo, la identificación visual y diferenciación de diferentes especies de Leishmania es, en la mayoría de los casos, una tarea imposible debido a similitudes morfológicas ${ }^{(14,15)}$. En este contexto, el uso de herramientas moleculares aplicadas a la identificación parasitológica ayuda a interpretar la real diversidad de agentes infecciosos, y así dirigir correctas acciones ante los agentes patológicos. Sin embargo, hay que ser cautos. Más estudios son necesarios para conocer si Leishmania (Viannia) lainsoni es una especie autóctona en Paraguay, y aún más, el grado de dispersión de la especie.

Ahora, con la inclusion de $L$. (V.) lainsoni a la fauna de parásitos de Paraguay, surgen más preguntas, tales como: ¿Cuántos casos realmente existen causados por esta especie? ¿Cuál es la distribución de los casos debidos a esta especie en el país? ¿Son los vectores de esta especie en Paraguay los mismos que en otros países? Respecto a esta última pregunta, el vector más importante para esta especie, es el flebótomo Lutzomyia ubiquitalis en Brazil ${ }^{(16,17)}$ y Lu. nuneztovari anglesi en Bolivia ${ }^{(18,19)}$, los cuales nunca fueron registrados en Paraguay.

Adicionalmente, sería importante identificar reservorios naturales. En otras áreas de Sudamérica, al norte de la localización del caso descrito aquí, el mamífero Cuniculus paca (Cuniculidae: Rodentia) fue identificado como reservorio natural ${ }^{(20,21)}$. Este roedor es abundante y de amplia distribución en Paraguay ${ }^{(21,22)}$ y ciertamente también puede ser el reservorio natural para $L$. (V.) lainsoni en el país. Son necesarios estudios adicionales para confirmar la presencia de este parásito en $C$. paca u otros animales en la naturaleza.

Leishmania (V.) Iainsoni fue registrada en los estados brasileros de Amapá, Pará y Rondônia ${ }^{(18,23)}$ y regiones sub-andinas de Bolivia y Perú ${ }^{(17,25,26)}$. Corrêa et al. ${ }^{(19)}$ ya mencionó que este parásito posee una amplia distribución en el neotrópico. Sin embargo, estudios posteriores mostraron que esta especie se distribuye principalmente por la región norte de Sudamérica alcanzando Ecuador, Surinam y la Guayana 
Francesa ${ }^{(27)}$, llegando parcialmente a Perú y Bolivia ${ }^{(17)}$ (Fig. 1). Esta especie parece estar asociada a bosques tropicales húmedos con una vasta distribución en la Ecorregión de la Amazonía. En este trabajo se brinda información sobre su presencia en Paraguay y por lo tanto la distribución real de Leishmania (V.) lainsoni podría ser más amplia hacia el sur, extendiendo así su rango de distribución conocido en unos 1,430 km hacia el sur (Fig. 1). El área donde el parásito fue encontrado en Paraguay corresponde a bosque templado húmedo, en el límite con el Chaco Húmedo. Un factor que hoy en día contribuye a la dispersión de las enfermedades son los fáciles medios para viajes de larga distancia, conducidos por el desarrollo de rutas de comunicación (fluviales, terrestres y aéreas).

El desarrollo de LC causada por $L$. (V.) lainsoni es básicamente el mismo que el observado por $L$. (Leishmania) amazonensis y L. (Viannia) braziliensis, y la diferencia más importante es que L. (V.) lainsoni no produce lesiones dérmicas ulceradas y nodulares ${ }^{(17,28)}$. Las lesiones de la piel del caso descrito aquí se ajustan con lo conocido para infecciones causadas por $L$. (V.) lainsoni. La evolución del caso no pudo continuarse debido a que la paciente volvió a su comunidad, sin Volver a tener contacto con el servicio asistencial.

Finalmente, es importante mencionar el valor de los estudios moleculares para la identificación taxonómica y su asociación con la severidad y características de la infección. Entonces, es altamente recomendable generar más análisis moleculares combinados con la vigilancia centinela normal en Paraguay, para apuntar a resolver algunas de las preguntas que surgieron en este estudio. En este sentido, el caso reportado aquí proviene de una zona endémica, comúnmente atribuida a $L$. (V.) braziliensis. Un mejor conocimiento de los agentes y riesgos de la leishmaniasis en Paraguay, ayudará a disminuir significativamente el número de casos en el país, o proveer una mejor asistencia a la población afectada.

\section{Agradecimientos}

Nuestro agradecimiento a Maria Teresa Barán, directora del SENEPA, por su constante apoyo y motivación en este estudio. También a Elisa Cupolillo por sugerencias realizadas sobre este trabajo. Finalmente, agradecemos al staff del Hospital Nacional de Itauguá por brindar asistencia médica a la paciente tratada.

\section{Referencias bibliográficas}

1. WHO. A global brief on vector-borne diseases. World Health Organization, Geneva; 2014. 54 p.

2. Garnham PCC. The genus Leishmania. Bull Wld Hlth Org. 1971; 44: 477-489.

3. Torres-Guerrero E, Quintanilla-Cedillo MR, Ruiz-Esmenjaud J, Arenas R. Leishmaniasis: a review. F1000 Res. 2017; 6: 750.

4. Pedroni E, Galeano R, Martínez B, Recalde D, Rojas A. Guía nacional de vigilancia y control de enfermedades. Sistema Nacional de Vigilancia Epidemiológica, Ministerio de Salud Pública y Bienestar Social: Asunción; 2015. 379 p.

5. Torales Ruotti M, Segovia L, Martínez Acosta N, Benítez Giménez I, Viveros C, Ruiz Díaz P, et al. Manual de Diagnóstico y Tratamiento de las Leishmaniasis. Servicio Nacional de Erradicación del Paludismo, Ministerio de Salud Pública y Bienestar Social: Asunción; 2018. 92 p

6. Oddone Costanzo R. Leishmaniosis visceral: a 101 años del primer caso diagnosticado en las Américas. Mem Inst Investig Cienc Salud 2012; 10(1): 100-104.

7. Brito A, Azulay D, Azulay R. Leishmaniosis y demás Protozoonosis de interés Dermatológico. En Azulay R, Azulay D . Azulay L. Azulay - Dermatología. 4a Ed. Rio de Janeiro: Guanabara - Koogan, 2006; p.415-422. 
8. Chena L,. Nara E, Canese A, Oddone R, Morán M, Russomando G. Caracterización de cepas de Leishmania, por medio de la técnica de PCR-RFLP de la región del Spliced Leader Miniexon (SLME), aisladas de humanos y caninos en Paraguay. Mem Inst Investig Cienc Salud, 2012; 10(1): 14-23.

9. Canese A. Leishmaniosis Tegumentaria en el Paraguay. Evolución de 22 años (1975-1996). Rev Parag Microb. 1998; 18(1): 25-29.

10. Caballero A, Guglielmone Pinto C, Rivelli Oddone V, Mendoza de Sanchez G, De Paula N, Almeida F, Roselino AM. Leishmaniasis cutánea atípica por L. infantum (Chagasi). An Fac Cienc Méd. 2013; 46(1): 61-67.

11. Requena JM, López MC, Jimenez-Ruiz A, de la Torre JC, Alonso C. A head-to-tail tandem organization of hsp70 genes in Trypanosoma cruzi. Nucleic Acid Res. 1988; 16(4): 1393-1406.

12. Graça GC, Volpini AC, Romero GA, Oliveira Neto MP, Hueb M, Porrozzi R, Coité MC, Cupolillo E. Development and validation of PCR-based assays for diagnosis of American cutaneous leishmaniasis and identification of the parasite species. Mem Inst Oswaldo Cruz. 2012; 107(5): 664-674.

13. Arévalo J, Ramirez L, Adaui V, Zimic M, Tulliano G, MirandaVerastegui C, Lazo M, Loayza-Muro R, De DS, Maurer A, Chappuis F, Dujardin J, Llanos-Cuentas A. Influence of Leishmania (Viannia) species on the response to antimonial treatment in patients with american tegumentary leishmaniasis. J Infec Dis. 2007; 195: 1846-1851.

14. Aviles H, Belli A, Armijos R, Monroy F, Harris E. PCR detection and identification of Leishmania parasites in clinical specimens in Ecuador: a comparison with classical diagnostic methods. J Parasitol. 1999; 85: 181-187.

15. Romero G, Guerra M, Paes M, Macedo VO. Comparison of cutaneous leishmaniasis due to Leishmania (Viannia) braziliensis and L. (V.) guyanensis in Brazil: therapeutic response to meglumine antimoniate. Am J Trop Med Hyg. 2001; 65: 456-465.

16. Lainson R, Shaw JJ, Souza AA, Silveira FT, Falqueto A. Further observations on Lutzomyia ubiquitalis (Psychodidae: Phlebotominae), the sandfly vector of Leishmania (Viannia) lainsoni. Mem Inst Oswaldo Cruz. 1992; 87: 437-439.

17. Scarpassa, VM, Alencar RB. Speciation in the Leishmania guyanensis Vector Lutzomyia umbratilis (Diptera: Psychodidae) from Northern Brazil — Implications for Epidemiology and Vector Control. In: Samie, A. (Ed.), An Overview of Tropical Diseases. IntechOpen, London. 2015; 83-105 pp.

18. Bastrenta B, Buitrago R, Vargas F, Le Pont F, Torrez M, Flores M, Mita N, Brenière SF. First evidence of transmission of Leishmania (Viannia) lainsoni in a sub-Andean region of Bolivia. Acta Trop. 2002; 83: 249-253.

19. Corrêa, JR, Brazil, RP, Soares, MJ. Leishmania (Viannia) lainsoni (Kinetoplastida: Trypanosomatidae), a divergent Leishmania of the Viannia subgenus - A mini review. Mem Inst Oswaldo Cruz. 2005; 100(6): 587-592.

20. Silveira F, Shaw J, Braga R, Ishikawa E. Dermal leismaniasis in the Amazon Region of Brazil: Leishmania (Viannia) lainsoni sp n, a new parasite from the State of Para. Mem Inst Oswaldo Cruz. 1982; 289292.

21. Dedet J. Leishmania et leishmanioses du continent américain. Annales de L’Institut Pasteur 1993; 4 (1): 3-25.

22. Canevari M, Vaccaro O. Guía de Mamíferos del Sur de América del Sur. Literature of Latin America, Buenos Aires. 2007; 413 p.

23. Saldívar S, Rojas V, Giménez D, et al. 2017. Los mamíferos amenazados del Paraguay. In: Saldívar, S., Rojas, V., Giménez, D. (eds.), Libro Rojo de los Mamíferos del Paraguay: especies amenazadas de extinción. Asociación Paraguaya de Mastozoología / Secretaría del Ambiente, Asunción. 27-33 pp.

24. Silveira FT, Souza AAA, Lainson R, Shaw JJ, Braga RR, Ishikawa EA. Cutaneous leishmaniasis in the Amazon region: natural infection of the sandfly Lutzomyia ubiquitalis (Psychodidae: Phlebotominae) by Leishmania (Viannia) lainsoni in Pará state, Brazil. Mem Inst Oswaldo Cruz. 1991; 86: 127-130. 
25. Lucas CM, Franke ED, Cachay MI, Tejada A, Cruz ME, Kreutzer RD, Barker DC, McCann SH, Watts DM. Geographic distribution and clinical description of leishmaniasis cases in Peru. Am J Trop Med Hyg. 1998; 59: 312-317.

26. Martinez E, Le Pont F, Mollinedo S, Cupolillo E. A first case of cutaneous leishmaniasis due to Leishmania (Viannia) Iainsoni in Bolivia. The Lancet. 2001; 95(4): 375-377.

27. Kato H, Bone A, Mimori T, Hashiguchi K, Shiguango GF, Gonzales SV, et al. First Human Cases of Leishmania (Viannia) lainsoni Infection and a Search for the Vector Sand Flies in Ecuador. PLoS Negl Trop Dis 10(5): e0004728.

28. Silveira FT, Moraes MAP, Lainson R, Shaw JJ. Leishmaniose cutânea experimental. III - Aspectos histopatológicos do comportamento evolutivo da lesão cutânea produzida em Cebus appela (Primates:Cebidae) por Leishmania (Viannia) lainsoni, L. (Viannia) braziliensis e L. (Leishmania) amazonensis. Rev Inst Med Trop São Paulo. 1990; 32: 387-394. 